\title{
A developmental study of visual search behavior , 2
}

\author{
ELEANOR J. GIBSON AND ALBERT YONAS
}

CORNELL UNIVERSITY

Children in second, fourth and sixth grades and college sophomores were compared on a visual search and scanning task under three experimental conditions. In Condition I, a single target letter was sought in a list of letters of low visual confusability. In Condition II, two target letters were sought but only one appeared in a given list. In Condition III, a single target letter was sought in a list of letters of high confusability. Search time decreased with age in all three tasks. Searching for two targets was no harder than searching for one. A highly confusable visual context increased search time at all age levels.

It has long been assumed that there are both qualitative and quantitative differences, developmentally, in attentive behavior; that there is increasing skill in filtering incoming information from the environment, selecting what has utility for the task and ignoring what is irrelevant. Systematically searching for a designated target, while ignoring distracting and unwanted background context, seemed to us to epitomize an activity in which strategies of selecting and processing information should vary with age. The present experiment investigated this question, employing a search and scanning task devised by Neisser (1964). The task requires searching for a specified target, such as a letter, embedded in a context of more or less similar items. If there are qualitative differences over age groups, these should show up as age and variable interactions when suitable parameters of the taskare manipulated.

Two variables were manipulated in the present experiment, number of targets sought at one time, and contextual confusability. Neisser et al (1963) have shown that adult Ss, after long practice, can search for as many as ten targets at once with little loss of speed. If parallel processing, or number of things that can be attended to at once, increases with age, we should expect an age interaction with increase in number of targets. On the other hand, if selective attention involves the learning of distinctive features of the set of items presented (i.e., those contrasting properties which make matching and differentiating possible), a highly confusable context might be relatively more difficult for children than for adults. Such a context would maximize the number of features shared by target and background items.

\section{METHOD}

Letters were chosen as stimuli. They were typed in lists of 30 four-letter strings, one string to a line. The typed list was $10 \mathrm{in.} \mathrm{long,} \mathrm{the} \mathrm{letters} 1 / 4 \mathrm{in}$. high. The list was fixed to a longer cardboard mounting which could be inserted in the display apparatus. The apparatus was a light-tight box with a slanting giass window (see Fig. 1). The glass was partially silvered so that the list was visible only when two lumiline lamps inside the box were turned on. Above the glass window, a small covered bulb was positioned so that a dot of light appeared on the mirror at the same point in space as the top line of the list when the lamps were on. This allowed $S$ to accommodate properly and start his scan precisely at the top of the list. The S's eyes were approximately $14 \mathrm{in.} \mathrm{from} \mathrm{the} \mathrm{mirror.} \mathrm{The} \mathrm{slope} \mathrm{of} \mathrm{the} \mathrm{mirror} \mathrm{(and}$ list beneath it) were such as to keep this distance approximately constant as the scan progressed downward.

The $\mathrm{S}$ was instructed to start at the top of the list and scan downward until he found the designated target letter, proceeding as rapidly as possible. He was urged to scan downward in order, without skipping any lines. He held a push button in his hand. When he was ready to begin scanning, he pressed the button which caused the list to appear and also started a Hunter clock-counter (calibrated in .001 sec.). The $\mathrm{S}$ immediately started scanning down the list. On finding the target letter, he pressed the button again. This stopped the clock and turned off the lamps. He was asked to indicate the approximate spot on the mirror where the target had

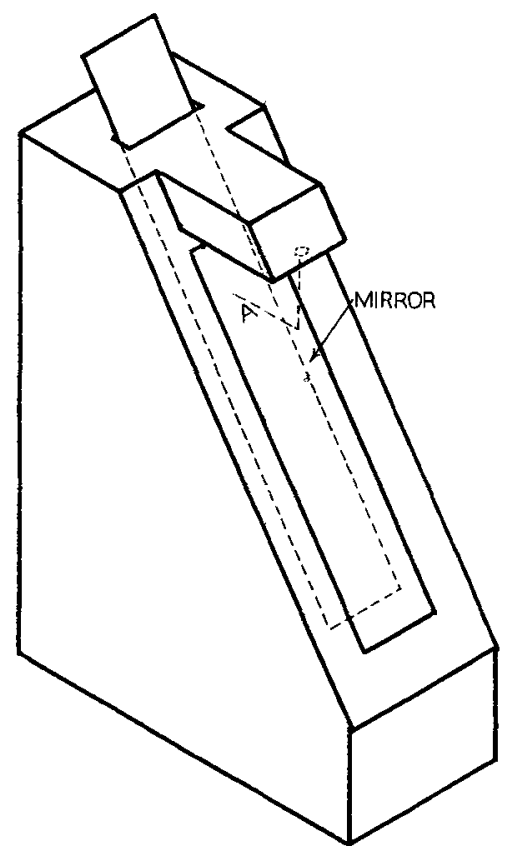

Fig. 1. Three-quarter view of the scanning apparatus. 
appeared in order to discourage guessing. The $\mathbf{E}$ then recorded the time and placed another list in the display box. If $\mathrm{S}$ had failed to find the target when he reached the bottom of the list, the list was setaside and rerun later. Five practice trials preceded the experiment proper.

The letters chosen were simplified capitals typed with an IBM sign-typewriter, because these are easy for a child to read and because this type had previously been analyzed for distinctive features and a confusion matrix was available (Gibson et al, 1964). The experimental design included three conditions.

Condition l was a single target search, with low contextual confusability. The $S$ was told to search for the letter G, which would appear only once on a list. The target letter remained the same for 20 trials. Its position in the 20 lists was randomized, with the restriction that it appeared equally often in four quadrants of the list (from top to bottom), never in the same line, and never in the first or the last line. In the practice trials, the target item did appear once in each of these lines to insure that a complete scan would be made. The six context letters in Condition I ( L, K, V, M, X, A) were ones which had seldom or never been confused with $G$ in the confusion matrix. They were randomly placed across and down the list, but appeared an equal number of times.

Condition II was a two-target search. The context was low-confusion again, but $S$ was told to look for either of two letters, G or R, only one of which would appear on any given list. He was not told which one. Each target appeared 10 times, randomly ordered through the 20 trials.

Condition III was again a single target task, but the context was highly confusable with the target letter $G$ (the letters $B, Q, C, J, S$, and $R$, which ranked highest in confusability with $G$ in the confusion matrix). There were 20 lists, with target position ordered as before.

The Ss were 72 children, 24 from each of three grades (second, fourth, and sixth), and a group of 12 college

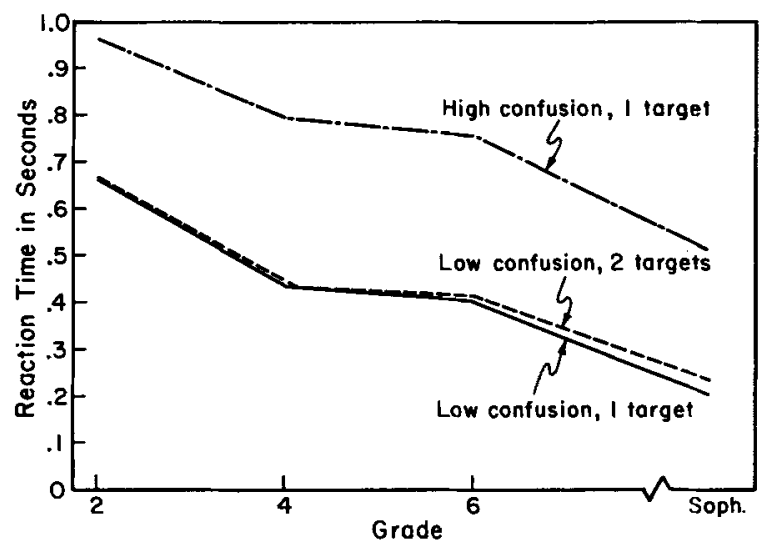

Effect of Target Number and Low and Hign-Confusion Context on Scanning Rote.

Fig. 2. Mean latencies for the three conditions of the experiment plotted by age group. sophomores who were fulfilling a requirement of the introductory course in psychology. There was an approximately equal number of each sex in each age group. Each $\mathrm{S}$ took part in two of the three conditions of the experiment, Condition I and either Condition II or Condition III. Condition I was always run first. Ss within a grade were paired by age, with one of the pair assigned to Condition II, the other to Condition III. Two rather than all three conditions were run because the children's time was limited to one-half hour. The experiment was run at a school ${ }^{3}$, during school hours. The college Ss were run in the laboratory.

\section{RESULTS}

In treating the results, the latency of detecting the target for each trial was transformed to take account of its position in the list. Theoretically, a linear relationship might be expected between target position and scanning rate. The expectation did not hold true for the first few list positions, however. Since a similar initial lag appeared for all three conditions, and for all age groups, all the transformed scores were included in the analysis. Means for the transformed scores are plotted by age group for the three conditions in Fig. 2. As expected, it is evident that speed of scan increased from second grade to college sophomores on all three conditions.

Three analyses of variance were performed on the transformed scores, one for the high-and low-confusion conditions, one for the one- and two-target conditions, and one for the two-target and high-confusion conditions. Grade differences were significant at the .05 level of significance or better in all conditions. The difference between the high- and low-confusion conditions was significant $(\mathrm{p}<.001)$, but the grade by conditions interaction was not. Context confusability was, it appears, equally damaging at all age levels. The difference between the one- and two-target conditions was not significant, nor was the interaction with grade. Two targets were searched for as efficiently as one, at all age levels. The high-confusion condition was significantly more difficult than the two-target condition $(p<.001)$.

Individual differences between Ss were marked, especially in the younger age groups. These differences interacted significantly with conditions, suggesting that strategies for different Ss vary with the task. This strategy difference was especially noticeable when younger Ss began the high-confusion task; some lowered the pace of scanning almost at once, while others were slower to do this and traded time for errors (missing the target). In general, however, the omissions reflected the same trends as the latencies.

\section{DISCUSSION}

Our expectation that increasing the number of things sought for at one time would be relatively more difficult for the younger Ss was not confirmed. The fact that the double-target search was no harder than the singletarget search confirms Neisser et al's (1963) finding, 
though searching for two targets is surely not as difficult as searching for ten. The Ss had the benefit of practice on Condition $i$ in the double target task, so performance might have looked poorer if practice had been balanced. But it seems clear that any difference, with two targets at least, would be negligible. One need not infer, however, that parallel processing is going on. Another explanation of the good performance in Condition II stems from analysis of distinctive features. The two target letters, $G$ and $R$, had in common a curve, but none of the context letters did. Optimal strategy, by feature processing, would be searching for one feature, a curve, which would differentiate the target from context letters. This possibility is at present being explored further in another experiment.

The effect of context on search speed is impressive. When the target letter is embedded in a context of letters containing a high percentage of common distinctive features (as these did) the search task is rendered far more difficult. However the features may be processed, sequentially or in parallel, discovery of the target takes much longer. It is not clear exactly how a simultaneous processing model would handle this finding, although a sequential model of visual discrimination, when a matching or discriminating judgment is required, would predict it. If discrimination involves tests of a feature list (the distinctive features of letters), then moving down the list until a difference is found would take much longer when the number of features common to the target and the context items is increased. That younger children should take longer than older ones or adults might be expected, since the feature set might be less well assembled and a possible hierarchical ordering of tests for greatest efficiency not yet optimized. The lack of an age-condition interaction, however, fails to support the possibility of a qualitative process change during development.

\section{References}

Gibson, E. J., Osser, H., Schiff, W., \& Smith, J. An analysis of critical features of letters, tested by a confusion matrix. In Final Report, Cooperative Research Project No. 639 with the Office of Education, $A$ basic research program on reading.

Neisser, U. Visual search. Scient. American, 1964, 210, 94-101. Neisser, U., Novick, R., \& Lazar, R. Searching for ten targets simultaneously. Percept. mot. Skills, 1963, 17, 955-961.

\section{Notes}

1. This research was supported by NIH Grant MH-07226-02.

2. We wish to express gratitude to Mr. Arthur McCaffrey for valuable assistance in running subjects.

3. Thanks are due Mr. Pastre, principal of the Cayuga Heights School, the teachers and the children.

(Received in the Editorial Office May 9, 1966.) 